\title{
Geometric Properties of the Adaptive Delaunay Tessellation
}

\author{
T. Bobach ${ }^{1}$, A. Constantiniu ${ }^{2}$, P. Steinmann ${ }^{2}$, G. Umlauf ${ }^{1}$ \\ ${ }^{1}$ Department of Computer Science, University of Kaiserslautern \\ \{bobach|umlauf\}@informatik.uni-kl.de \\ ${ }^{2}$ Institute of Applied Mechanics, University of Erlangen-Nuremberg, Germany \\ \{constantiniu|paul.steinmann\}@ltm.uni-erlangen.de
}

\begin{abstract}
Recently, the Adaptive Delaunay Tessellation (ADT) was introduced in the context of computational mechanics as a tool to support Voronoi-based nodal integration schemes in the finite element method. While focusing on applications in mechanical engineering, the former presentation lacked rigorous proofs for the claimed geometric properties of the ADT necessary for the computation of the nodal integration scheme. This paper gives pending proofs for the three main claims which are uniqueness of the ADT, connectedness of the ADT, and coverage of the Voronoi tiles by adjacent ADT tiles. Furthermore, this paper provides a critical assessment of the ADT for arbitrary point sets.
\end{abstract}

\section{Introduction}

One building block in finite element analysis is the background tessellation of a spatial domain, where requirements vary with the applications at hand. The corresponding field of mesh generation traditionally focuses on the generation of both vertex positions and connectivity, starting from a description of the domain boundary. Vertices are placed heuristically to adaptively sample local features and maintain a good shape of the resulting elements, although the discussion about the optimal shape is not settled even for triangles [1]. The tessellation can be done in ad-hoc constructions [2], iteratively [3], or based on guidance fields $[4,5]$ to name a few. In the rare occasion of a predetermined set of vertices, the choice of methods is generally limited to the constrained Delaunay triangulation or other, data-dependent, triangulations [6].

In [7], a novel tessellation technique, called Adaptive Delaunay Tessellation $(\mathrm{ADT})$, was introduced in the context of computational mechanics as a tool to support Voronoi-based nodal integration schemes in the Finite Element Method. Its main contribution is a simplified access to the element structure that supports nodal integration schemes based on a robust and unique transformation of the Delaunay triangulation. While focusing on the applications in linear elasticity, the presentation in [7] lacks rigorous proofs for the geometric properties of the ADT which are uniqueness of the ADT, connectedness of the ADT, and coverage of the Voronoi tiles by adjacent ADT tiles. These properties are essential for the 
computation of the nodal integration scheme. The pending proofs for these main claims are given in this paper.

We start with a motivation for the ADT and introduce some notation used in the rest of this paper in Section 2. Uniqueness and connectedness of the ADT are stated and proved in Sections 3.1 and 3.2. Section 3.3 introduces an alternative characterization of the ADT to simplify the proof of the last important property in Section 3.4 on the coverage of the Voronoi tile of a vertex by ADT elements adjacent to it. We discuss the ADT in Section 4 and conclude in Section 5.

\subsection{Linear Elasticity and Nodal Integration in the Finite Element Method}

The Finite Element Method encompasses a wide range of applications to model complex continuous phenomena based on a description composed of many small parts, called finite elements, which allow a structurally simpler definition of the phenomenon. In the field of computational mechanics, which is the main target of the ADT, one of the most basic and well-studied problems is that of linear elasticity, from which we sketch the key aspects in a simplified form.

The Finite Element Method typically requires the evaluation of the integral

$$
\int_{\Omega} \nabla \psi \cdot f
$$

for the so-called test function $\psi$, and some function $f$. In general, $\psi$ is an interpolant that is defined piecewise over the elements $\Omega_{i}$ of the domain. This allows the decomposition of the global integral into a sum of local integrals,

$$
\sum_{i} \int_{\Omega_{i}} \nabla \psi \cdot f
$$

This is the heart of the Finite Element Method and allows the numerical assessment of the problem by concentrating on its local reformulation for each $\Omega_{i}$ and the associated local interpolant $\psi_{i}=\left.\psi\right|_{\Omega_{i}}$. Disadvantages of the element-based approach are the requirement of derivatives of the test function $\psi$, and numerical problems if the elements $\Omega_{i}$ are badly shaped, as discussed in [1].

One remedy for these problems is to turn to meshless methods, for which the discretization focuses on vertices rather than elements. One meshless approach based on natural neighbor coordinates has been proposed in [8]. Another meshless technique is nodal integration, as introduced in [9]. Here, an alternative tessellation of the domain is considered, where the vertices $\mathbf{v}_{j}$ are enclosed by polygons $\mathcal{T}_{j}$, with $\Omega=\bigcup_{j} \mathcal{T}_{j}$ and the above integral becomes

$$
\sum_{j} \int_{\mathcal{T}_{j}} \nabla \psi \cdot f
$$

assigning a part of the integral to each node $\mathbf{v}_{j}$ rather than to an element $\Omega_{i}$. The canonical choice for this tessellation is the Voronoi diagram of the vertices 
$\mathbf{v}_{j}$ of the background tessellation, restricted to $\Omega$. The application of Stokes' theorem allows to write

$$
\int_{\mathcal{T}_{j}} \nabla \psi \cdot f=\int_{\partial \mathcal{T}_{j}} \mathbf{n} \cdot \psi \cdot f
$$

where $\mathbf{n}$ is the outward pointing unit vector. By turning the area integral into a path integral, no derivatives of $\psi$ are required, but now the local integration domains $\mathcal{T}_{i}$ do no longer correspond to the elements $\Omega_{j}$ of the background tessellation. Therefore, the computation of the path integral for each node can no longer be carried out on a per-element basis, but requires the evaluation of the test function $\psi$ at arbitrary positions along $\partial \mathcal{T}_{j}$.

\subsection{Voronoi Tile Coverage in the ADT}

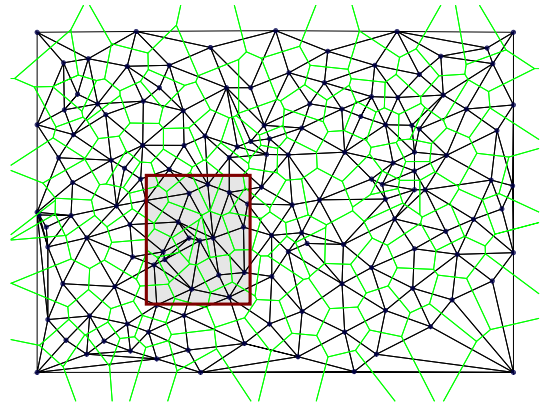

(a)

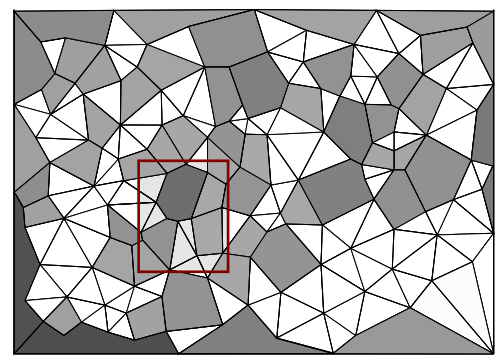

(c)

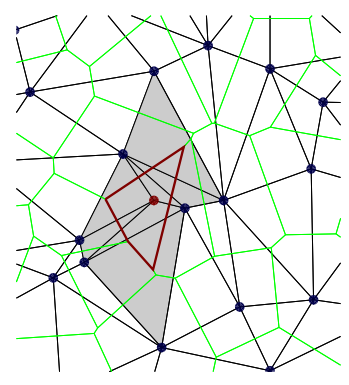

(b)

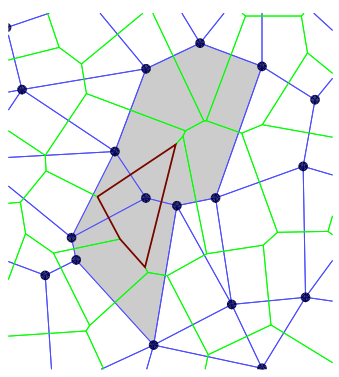

(d)

Fig. 1. Example of an ADT tessellation. The point cloud with its Voronoi diagram in green and the Delaunay triangulation in black is shown in (a). The closeup of a node with shaded triangles depicting the necessary cover for its Voronoi tile is shown in (b). The corresponding ADT with polygons shaded according to valence is shown in (c). In the closeup (d), it is apparent that the adjacent ADT polygons cover the Voronoi tile.

By covering the Voronoi tile of a vertex with a minimal set of adjacent polygons, the computational assessment of Voronoi-based nodal integration is greatly 
simplified. Figure 1 shows a set of scattered points covering a rectangular domain. The boundary of the Voronoi tile shown in Figure 1(b) is a convex polygon that intersects certain triangles in the Delaunay triangulation of the point set, which are not necessarily adjacent to the Voronoi site. The ADT, on the other hand, consists of polygons that are big enough to cover the whole Voronoi tile. Thus, by traversing the polygons adjacent to a vertex, one has access to an area that completely covers the Voronoi tile of that vertex.

\section{The Adaptive Delaunay Tessellation}

Before presenting the main findings, we introduce the necessary notation and repeat some important results.

We consider the canonical open ball topology on $\mathbb{R}^{2}$ and denote by $[\Omega]$ the topological closure of a set $\Omega$, by ] $\Omega$ [ its topological interior, and by $\partial \Omega:=$ $[\Omega] \backslash] \Omega\left[\right.$ its topological boundary. For a set $\mathbf{P} \subset \mathbb{R}^{2}$ of points, we denote by $\mathscr{C}(\mathbf{P})=] \mathscr{C}(\mathbf{P})[\cup \partial \mathscr{C}(\mathbf{P})$ the convex hull of $\mathbf{P}$.

We denote by $(F, E)$ a partition of $\mathscr{C}(\mathbf{P})$ into polygonal faces $f \in F$ that join along edges $e \in E$. By $\operatorname{DeL}(\mathbf{P}):=\left(F_{\mathrm{DEL}}, E_{\mathrm{DEL}}\right)$ we refer to a Delaunay triangulation of $\mathbf{P}$, composed of triangular faces $f \in F_{\mathrm{DEL}} \subset \mathbf{P}^{3}$ and edges $e \in E_{\text {DeL }} \subset \mathbf{P}^{2}$. We will use $f$ and $e$ to both denote the set of vertices from $\mathbf{P}$ and their geometric realizations. Two faces $f_{1}$ and $f_{2}$ with $f_{1} \cap f_{2}=e \in E_{\mathrm{DEL}}$ are called neighbors with common edge $e$. We consider angles greater or equal $\pi / 2$ as obtuse and a triangle with an obtuse interior angle is called obtuse. The longest edge of an obtuse triangle $f$ is opposite to its obtuse angle and will be denoted by $e_{f}^{>}=\arg \max _{e \in f}|e|$. Whenever the term $e_{f}^{>}$is used, $f$ implicitly represents an obtuse triangle. For a triangle $f \in F_{\text {DeL }}$ we denote by $\operatorname{cc}(f)$ its circumcenter. Note that a triangle $f$ is obtuse if it does not contain $\operatorname{cc}(f)$ in its interior and that $\operatorname{cc}(f)$ lies on the opposite side of $e^{>}$as the obtuse angle.

We denote by $G=(\mathbf{P}, E)$, a graph over $\mathbf{P}$, where $\mathbf{P}$ represents the set of graph vertices and $E \subset \mathbf{P}^{2}$ the set of of edges. A sequence of vertices $\rho=\left(\mathbf{p}_{1}, \ldots, \mathbf{p}_{n}\right)$, $\left(\mathbf{p}_{i}, \mathbf{p}_{i+1}\right) \in E$, is a path of length $|\rho|=n$ if none of its edges appears more than once, i.e., edges are mutually distinct. A path is called closed if $\left(\mathbf{p}_{n}, \mathbf{p}_{1}\right) \in E$. We assume a path to be free of loops of length one, i.e., edges $\left(\mathbf{p}_{i}, \mathbf{p}_{i}\right)$. The Delaunay triangulation itself represents an undirected, connected graph $G_{\mathrm{DEL}}=\left(\mathbf{P}, E_{\mathrm{DEL}}\right)$.

The Voronoi diagram of $\mathbf{P}$ is the partition of $\mathbb{R}^{2}$ into convex polygons $\mathcal{T}_{\mathbf{p}}$, called Voronoi tiles of $\mathbf{p}$, such that every point in $\mathcal{T}_{\mathbf{p}}$ is not farther from $\mathbf{p}$ than from any other vertex in $\mathbf{P}$. For each vertex $\mathbf{p} \in \mathbf{P} \backslash \partial \mathscr{C}(\mathbf{P})$, its Voronoi tile is equal to $\mathcal{T}_{\mathbf{p}}=\mathscr{C}\left(\left\{\mathrm{cc}(f) \mid f \in F_{\mathrm{DeL}}, \mathbf{p} \in f\right\}\right)$, and we also call $\mathbf{p}$ a Voronoi site.

With these definitions the Adaptive Delaunay Tessellation is defined as in [7]:

Definition 1 (Adaptive Delaunay Tessellation). Let $\mathbf{P} \subset \mathbb{R}^{2}, \operatorname{Del}(\mathbf{P})=$ $\left(F_{\mathrm{DeL}}, E_{\mathrm{DeL}}\right)$ and

$$
E^{>}=\left\{e_{f}^{>} \mid f \in F_{\mathrm{DEL}} \wedge e_{f}^{>} \not \subset \partial \mathscr{C}(\mathbf{P})\right\} .
$$


The tessellation of $\mathscr{C}(\mathbf{P})$ represented by $\left(F_{\mathrm{ADT}}, E_{\mathrm{DEL}} \backslash E^{>}\right)$, where $F_{\mathrm{ADT}}$ is the set of faces generated by merging triangles with common edges in $E^{>}$, is called the Adaptive Delaunay Tessellation $\operatorname{ADT}(\mathbf{P})$.

Thus, the ADT of a point set is the result of removing from a Delaunay triangulation of $\mathbf{P}$ of each obtuse triangle the longest edge, if this is not a boundary edge. Since no new edges are generated in $\operatorname{ADT}(\mathbf{P})$, each triangle $f \in F_{\mathrm{DEL}}$ is part of some polygon $g \in F_{\mathrm{ADT}}$, which we denote by $\mathscr{A}(f)=g \supset f \in F_{\mathrm{DEL}}$.

\section{Geometric Properties of the ADT}

In this section we will discuss some of the geometric properties of $\operatorname{ADT}(\mathbf{P})$, i.e., uniqueness, connectedness, and the inclusion of Voronoi tiles. The last property states that the Voronoi tile of each $\mathbf{p} \in \mathbf{P}$ is contained in the union of $\mathbf{p}$ 's adjacent faces from $\operatorname{ADT}(\mathbf{P})$.

\subsection{Uniqueness of the ADT}

Proposition 1 (Uniqueness). For any non-collinear set of points $\mathbf{P} \subset \mathbb{R}^{2}$, $\operatorname{ADT}(\mathbf{P})$ exists and is unique.

Proof. We will show that $E^{>}$contains all edges of $E_{\text {DeL }}$ that are non-unique.

For any non-collinear point set $\mathbf{P}, \operatorname{DeL}(\mathbf{P})$ exists, and is non-unique, if there are at least $n \geq 4$ points $\mathbf{p}_{1}, \ldots, \mathbf{p}_{n}$ with a common empty circumcircle $\mathbf{C}$ with circumcenter $\mathbf{c}$, i.e., $\mathbf{P} \cap] \mathbf{C}[=\emptyset$. The convex hull of these points can be triangulated arbitrarily by edges $e_{1}, \ldots, e_{n-3}$ without violating the Delaunay condition, see Figure 2(a). Every $e_{i}$ connects two points not neighboring on the circumcircle and belongs to two triangles of the triangulation of $\mathscr{C}\left(\left\{\mathbf{p}_{1}, \ldots, \mathbf{p}_{n}\right\}\right)$. Because there is at most one non-obtuse triangle containing the circumcenter $\mathbf{c}$, every edge $e_{i}$ belong to at least one obtuse triangle. Furthermore, every edge $e_{i}$ is the longest edge of the obtuse triangle that lies on the opposite side of $e_{i}$ with respect to $\mathbf{c}$. Thus, $e_{i} \in E^{>}$.

In case $\mathbf{c}$ lies on an edge, say $e_{j}$, then $e_{j} \in E^{>}$, because at least one of its triangles is rectangular, see Figure 2(b). So, all $e_{i}$ are in $E^{>}$, such that $E_{\mathrm{DEL}} \backslash E^{>}$ is unique.

Remark 1. If in the above setting the common edge $e$ of two triangles is passing through the common circumcenter, both triangles are rectangular, i.e., obtuse, and $e$ is removed.

\subsection{Connectedness of the ADT}

Before stating the second geometric property, we introduce some results used in its proof. For every obtuse triangle $f$ with longest edge $e_{f}^{>}$and neighbor $f^{\prime}$, i.e., $f \cap f^{\prime}=e_{f}^{>}$, we use

$$
N(f):=f^{\prime}
$$




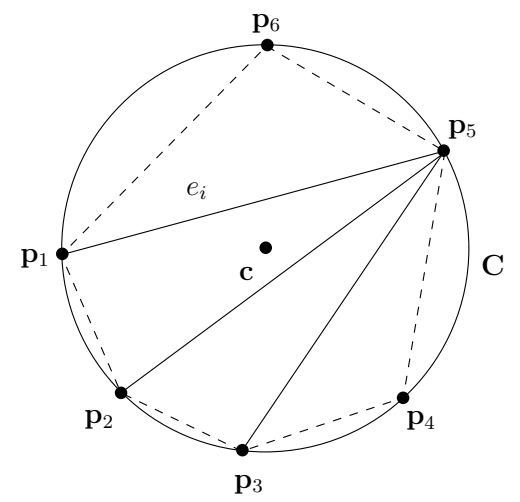

(a)

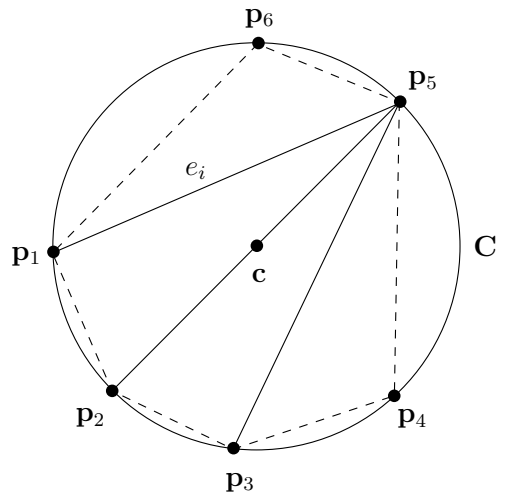

(b)

Fig. 2. Points $\mathbf{p}_{1}, \ldots, \mathbf{p}_{n}$ on a common circumcircle $\mathbf{C}$ with center $\mathbf{c}$ inside a triangle (a) or on an edge $e_{j}(\mathrm{~b})$.

to denote neighbor $f^{\prime}$ of $f$ opposite to the obtuse angle. This relation imposes a sub-graph of the dual Delaunay triangulation on the faces of $\operatorname{DEL}(\mathbf{P})$. For non-obtuse triangles or if $e_{f}^{>} \subset \partial \mathscr{C}(\mathbf{P})$, we set $N(f)=\emptyset$, and $N(\emptyset)=\emptyset$. This induces the directed graph

$$
\begin{aligned}
& \vec{G}_{\mathrm{DEL}}:=\left(F_{\mathrm{DEL}}, \vec{E}_{\mathrm{DEL}}\right), \\
& \vec{E}_{\mathrm{DEL}}:=\left\{(f, N(f)) \mid f \in F_{\mathrm{DeL}} \wedge N(f) \neq \emptyset\right\} .
\end{aligned}
$$

Each $\vec{e} \in \vec{E}_{\text {DeL }}$ is associated with one Delaunay edge $e \in E^{>}$.

In the proof we use the following lemma, which implies that the lengths of the longest edges of obtuse triangles grow along a path in $\vec{G}_{\text {DeL }}$.

Lemma 1. For $f \in F_{\mathrm{DeL}}$ with $N(f) \neq \emptyset$,

$$
e_{f}^{>} \neq e_{N(f)}^{>} \Rightarrow\left|e_{f}^{>}\right|<\left|e_{N(f)}^{>}\right| .
$$

Proof. We first show that there cannot be obtuse angles facing the same edge unless they are both right angles. Consider Figure 3(a) and assume $\triangle A B C$ and $\triangle C D A$ with common edge $\overline{A C}$ are triangles in a Delaunay triangulation. Let $D^{\prime}$ be a point on the circumcircle $\mathbf{C}$ of $\triangle A B C$ right of $\overline{A C}$. Since $\square A B C D^{\prime}$ is a circular, simple quadrilateral, $\angle A B C+\angle C D^{\prime} A=\pi$. By the Delaunay criterion, $D$ must either be on or outside $\mathbf{C}$, and comparison to the inscribed angle yields $\angle C D A \leq \angle C D^{\prime} A=\pi-\angle A B C$. The only obtuse angles that yield equality are $\angle C D A=\angle A B C=\pi / 2$, in which case $e_{f}^{>}=e_{N(f)}^{>}$.

In all other cases, i.e., $e_{f}^{>} \neq e_{N(f)}^{>}$, we find $e_{f}^{>}$adjacent to the obtuse angle in $N(f)$ and therefore shorter than $e_{N(f)}^{>}$, see Figure $3(\mathrm{~b})$. This yields (2). 


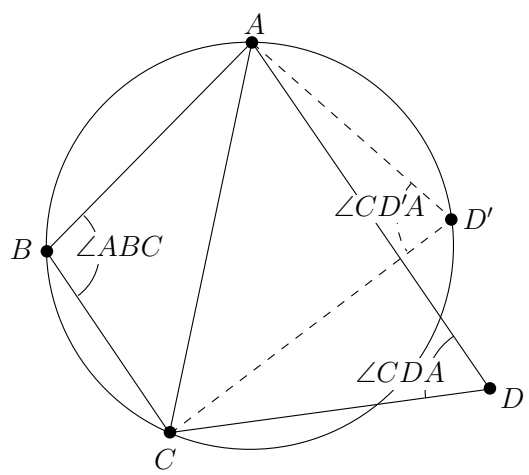

(a)

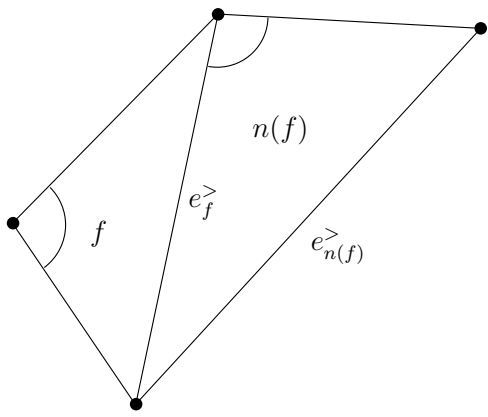

(b)

Fig. 3. (a) Two Delaunay triangles with the circumcircle of $\triangle A B C$. (b) $\left|e_{f}^{>}\right|<\left|e_{N(f)}^{>}\right|$.

Now we can state the result which guarantees that the transformation from Delaunay to adaptive Delaunay does not lead to unconnected elements such as orphaned vertices or even disconnected sub-graphs.

Proposition 2 (Connectedness). For $\operatorname{ADT}(\mathbf{P})=\left(F_{\mathrm{ADT}}, E_{\mathrm{ADT}}\right)$ the graph $G_{\mathrm{ADT}}=\left(\mathbf{P}, E_{\mathrm{ADT}}\right)$ is connected.

Proof. $G_{\mathrm{ADT}}$ is a sub-graph of $G_{\mathrm{DeL}}$, and $G_{\mathrm{DeL}}$ is connected. An edge of $G_{\mathrm{DeL}}$ is not in $G_{\mathrm{ADT}}$ if and only if its dual edge is in $\vec{G}_{\mathrm{DEL}}$. The smallest subset of $G_{\mathrm{ADT}}$ which can be disconnected consists of a single vertex. Boundary vertices cannot be disconnected, since the duals of boundary edges are by definition not in $\vec{G}_{\mathrm{DEL}}$. An inner vertex in a Delaunay triangulation has a minimum valence of three, which means it cannot be disconnected in $G_{\mathrm{ADT}}$ unless there is a closed path of length greater or equal to three in $\vec{G}_{\mathrm{DeL}}$.

We show that a path $\rho$ can only be closed if it has length $|\rho|=2$. Let without loss of generality $\rho=\left(f_{1}, \ldots, f_{n}\right)$ be a path in $\vec{G}_{\text {DEL }}$, i.e., $f_{i+1}=N\left(f_{i}\right)$. First assume $e_{f_{i}}^{>} \neq e_{f_{i+1}}^{>}, 1 \leq i<n$. With (2) we get

$$
\left|e_{f_{1}}^{>}\right|<\left|e_{f_{2}}^{>}\right|<\cdots<\left|e_{f_{n}}^{>}\right| .
$$

If $\rho$ were closed, $N\left(f_{n}\right)=f_{1}$ and $e_{f_{n}}^{>}=f_{1} \cap f_{n}$ is both an edge of $f_{1}$ and larger than $e_{f_{1}}^{>}$. Since this is a contradiction to $e_{f_{1}}^{>}$being the largest edge in $f_{1}, \rho$ cannot be closed.

Now assume there is an $i$ such that $e_{f_{i}}^{>}=e_{f_{i+1}}^{>}$. Since elements in $\rho$ are mutually distinct and $N\left(f_{i+1}\right)=f_{i}, f_{i+1}$ is the last element in $\rho$ and $n=i+1$. For $\rho$ to be closed, $N\left(f_{n}\right)=f_{1}$ must hold. Thus

$$
N\left(f_{n}\right)=N\left(f_{i+1}\right)=f_{i}=f_{1},
$$

which leaves $i=1$ and $n=2$, and $\rho$ can only be closed if it has length 2 . Since the longest closed path in $\vec{G}_{\mathrm{DEL}}$ has length $|\rho|=2, G_{\mathrm{ADT}}$ is connected. 
Remark 2 (Implementation). From the above reasoning it appears that numerical instabilities might flip the inequalities and result in ambiguous or even inconsistent results. However, the only setting where the inequalities in (2) are "only just" fulfilled arises with co-circular points, where the uniqueness of the ADT should be forced by allowing an epsilon threshold.

\subsection{An Alternative Characterization for the ADT}

In the following, we make use of an alternative characterization of the ADT, involving the following construction, see Figures 4(a) and 4(b).

Definition 2 (Polygonal Extension $P E(f)$ ). For each triangular face $f$, we define

$$
P E(f)=] \mathscr{C}(f \cup\{\mathrm{cc}(f)\})[\cup\{\mathrm{cc}(f)\}
$$

as the Polygonal Extension of $f$, where $\operatorname{cc}(f)$ denotes the circumcenter of $f$.

Note that $P E(f)$ does not contain its boundary except for the circumcenter itself, which is necessary to cover the case of rectangular triangles, as we will see later. We know that if $f$ is non-obtuse, we get

$$
\operatorname{cc}(f) \in] f[, \quad \text { and } \quad] f[=P E(f) .
$$

If $f$ is obtuse, then $P E(f)$ extends over $e_{f}^{>}$, and

$$
\operatorname{cc}(f) \notin] f\left[, \quad \text { and } \quad e_{f}^{>} \cap P E(f) \neq \emptyset .\right.
$$

The following lemma is required in the proof of Proposition 3.

Lemma 2. Let $f$ be an obtuse triangle and $f^{\prime}=N(f) \neq \emptyset$. Then,

$$
P E(f) \backslash f \subset P E\left(f^{\prime}\right) .
$$

Proof. Consider triangles $f=\triangle A B C, f^{\prime}=\triangle A C D$ and their circumcenters $\operatorname{cc}(f), \operatorname{cc}\left(f^{\prime}\right)$ in Figure 4(c). It is sufficient to show that the excess area $P E(f) \backslash f$ given by $] \mathscr{C}(A, C, \operatorname{cc}(f))$ [ is already contained in a subset of $P E\left(f^{\prime}\right)$ given by ] $\mathscr{C}\left(A, C, \operatorname{cc}\left(f^{\prime}\right)\right)[$, as illustrated in Figure 4(d).

By definition, $f$ shares the $e_{f}^{>}=\overline{A C}$ with its neighbor $f^{\prime}=N(f)$. The circumcenters $\operatorname{cc}(f)$ and $\operatorname{cc}\left(f^{\prime}\right)$ lie on the perpendicular bisector of $e_{f}^{>}$on the same side of $e_{f}^{>}$as $D$. By the Delaunay empty circumcircle criterion, $D$ is outside the circumcircle of $f$, and on the side of $e_{f}^{>}$opposite to $B$, thus the distance of $\operatorname{cc}\left(f^{\prime}\right)$ to $e_{f}^{>}$is greater or equal to that of $\operatorname{cc}(f)$. Consequently,

$$
\mathscr{C}\left(\{A, C, \operatorname{cc}(f)\} \subset \mathscr{C}\left(\left\{A, C, \operatorname{cc}\left(f^{\prime}\right)\right\}\right) \subset \mathscr{C}\left(\left\{A, C, \operatorname{cc}\left(f^{\prime}\right), D\right\}\right) .\right.
$$

This relation also applies to the interior of these sets, which means

$$
\begin{aligned}
P E(f) \backslash f & =] \mathscr{C}(\{A, C, \operatorname{cc}(f)\})[ \\
& \subset] \mathscr{C}\left(\left\{A, C, \operatorname{cc}\left(f^{\prime}\right), D\right\}\right)\left[\cup\left\{\operatorname{cc}\left(f^{\prime}\right)\right\}=P E\left(f^{\prime}\right),\right.
\end{aligned}
$$

see Figure $4(\mathrm{~d})$. Note that in case of a rectangular triangle, the circumcenter lies on its obtuse edge, and $] \mathscr{C}(\{A, C, \operatorname{cc}(f)\})\left[=\emptyset \subset P E\left(f^{\prime}\right)\right.$ for arbitrary $f^{\prime}$. 


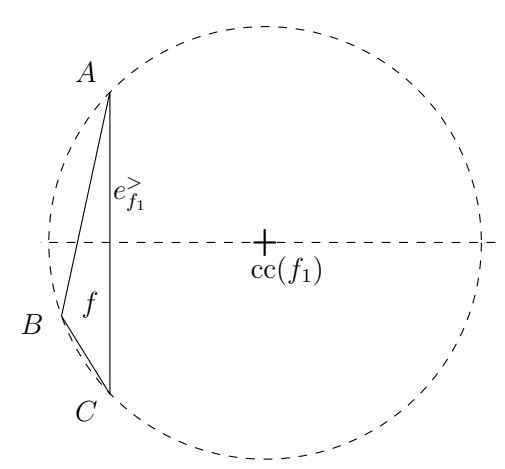

(a) Triangle $f$ and its circumcenter $\operatorname{cc}(f)$.

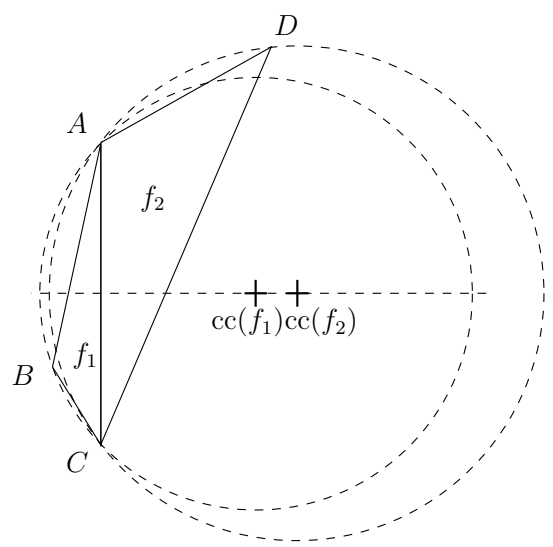

(c) Triangles $f$ and $f^{\prime}$.

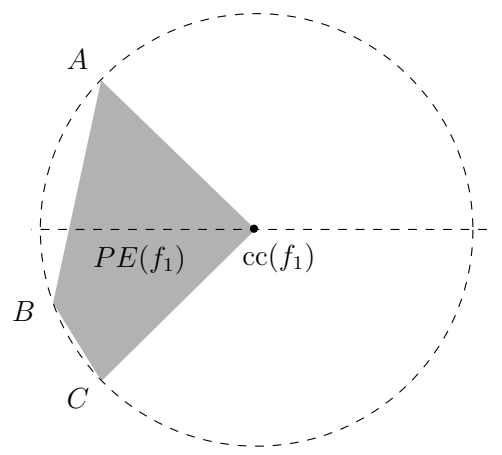

(b) Polygonal extension $P E(f)$.

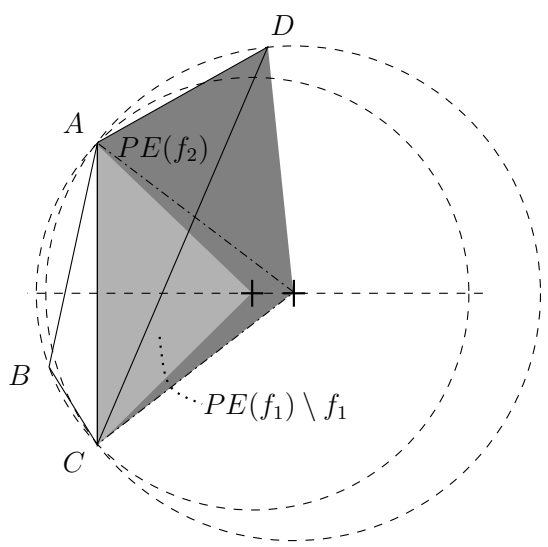

(d) $P E(f) \backslash f$ in light gray and $P E\left(f^{\prime}\right)$.

Fig. 4. Geometric interpretation of the polygonal extension $P E(f)$.

Lemma 2 implies an equivalent characterization for the set of edges $E^{>}$that are removed when transforming a Delaunay triangulation into an ADT.

Proposition 3. Let $\operatorname{DeL}(\mathbf{P})=\left(F_{\mathrm{DeL}}, E_{\mathrm{DeL}}\right)$ and $E^{>}$as in Definition 1. Then

$$
\left.e \in E^{>} \quad \Leftrightarrow \quad \exists f \in F_{\mathrm{DEL}}: e \cap P E(f) \cap\right] \mathscr{C}(\mathbf{P})[\neq \emptyset .
$$

So, an adaptive Delaunay tessellation can also be created by merging all triangles, whose polygonal extensions intersect, into polygons.

Proof. " $\Rightarrow "$ : If $e \in E^{>}$, then $e=e_{f}^{>}$for some obtuse $f \in F_{\mathrm{DEL}}$, and $e \cap] \mathscr{C}(\mathbf{P})[\neq \emptyset$. Then, (4) yields also $e \cap P E(f) \cap] \mathscr{C}(\mathbf{P})[\neq \emptyset$.

$" \Leftarrow "$ : Let $e \cap P E(f) \cap] \mathscr{C}(\mathbf{P})\left[\neq \emptyset\right.$ for an $f \in F_{\mathrm{DEL}}$. If $e$ is an edge of $f$ then $e=e_{f}^{>}$and $e \in E^{>}$. Otherwise, $e \neq e_{f}^{>}$, and $e \cap P E(f) \backslash f \neq \emptyset$. By Lemma 2 there exists an obtuse triangle $f^{\prime}$ such that $e \cap P E\left(f^{\prime}\right) \neq \emptyset$. This argument 
applies iteratively until $e$ is an edge of $f^{\prime}$. Since $\left|F_{\text {DeL }}\right|$ is finite and the subset relation in Lemma 2 is strict, this iteration terminates with a $f^{\prime} \in F_{\text {DeL }}$ such that $e$ is an edge of $f^{\prime}$. The final $f^{\prime}$ exists, otherwise $e$ would intersect the interior of a triangle and $\operatorname{DEL}(\mathbf{P})$ was no triangulation.

The relation of triangles, polygonal extensions and ADT faces is captured in the following result.

Proposition 4. For every $f \in F_{\mathrm{DEL}}$,

$$
f \subseteq[P E(f)] \cap \mathscr{C}(\mathbf{P}) \subseteq \mathscr{A}(f) .
$$

Proof. The left inclusion is true by construction of $P E(f)$ and $f \subseteq \mathscr{C}(\mathbf{P})$.

The right inclusion is more difficult to show. If a triangle $f$ is non-obtuse, or rectangular, $\operatorname{cc}(f) \in f$ and $[P E(f)]=f \subseteq \mathscr{A}(f)$. In all other cases, $f$ is obtuse and $\operatorname{cc}(f) \notin f$. If $e_{f}^{>}$is on the boundary of $\mathscr{C}(\mathbf{P})$, then $[P E(f)] \cap \mathscr{C}(\mathbf{P})=f \subseteq$ $\mathscr{A}(f)$. Otherwise, $f$ is obtuse and there is a triangle $f^{\prime}=N(f)$ such that

$$
\begin{aligned}
& P E(f) \backslash f \subseteq P E\left(f^{\prime}\right) \quad \text { by }(5), \\
& \Rightarrow \quad P E(f) \subseteq f \cup P E\left(f^{\prime}\right), \\
& \Rightarrow \quad[P E(f)] \subseteq f \cup\left[P E\left(f^{\prime}\right)\right], \\
& \Rightarrow \quad[P E(f)] \cap \mathscr{C}(\mathbf{P}) \subseteq f \cup\left[P E\left(f^{\prime}\right)\right] \cap \mathscr{C}(\mathbf{P}) .
\end{aligned}
$$

Since $\mathscr{A}(f)=\mathscr{A}\left(f^{\prime}\right)$, the claim holds if we can prove $\left[P E\left(f^{\prime}\right)\right] \cap \mathscr{C}(\mathbf{P}) \subseteq \mathscr{A}\left(f^{\prime}\right)$. The argument applies repeatedly until $f^{\prime}$ is non-obtuse, rectangular, or has its obtuse edge on the boundary of $\mathscr{C}(\mathbf{P})$ following a path $\rho$ in $\vec{G}_{\text {DEL }}$. In the proof for Proposition 2 we showed that every path $\rho$ ends in a triangle $f$ that is either non-obtuse, has $e_{f}^{>}$on the convex hull, or in a loop consisting of two rectangular triangles. Therefore, the repeated application of the argument terminates and the claim is proved.

An important observation following immediately from the above Proposition is given in the following corollary:

Corollary 1. For every $f \in F_{\mathrm{DeL}}$ with $\operatorname{cc}(f) \in \mathscr{C}(\mathbf{P}): \operatorname{cc}(f) \in \mathscr{A}(f)$.

\subsection{Coverage of Voronoi Tiles}

The last property we are going to prove is the coverage of a vertex' Voronoi tile by its adjacent ADT tiles, as illustrated in Figure 5(a). With this property, the adjacency information contained in the ADT is sufficient to access the whole area covered by the Voronoi cell of a vertex.

Proposition 5 (Inclusion of Voronoi Tile). If $\mathcal{T}_{\mathbf{p}}$ denotes the Voronoi tile of $\mathbf{p} \in \mathbf{P}$, then

$$
\mathcal{T}_{\mathbf{p}} \cap \mathscr{C}(\mathbf{P}) \subset \bigcup_{\substack{g \in F_{\mathrm{ADT}} \\ g \ni \mathbf{p}}} g .
$$




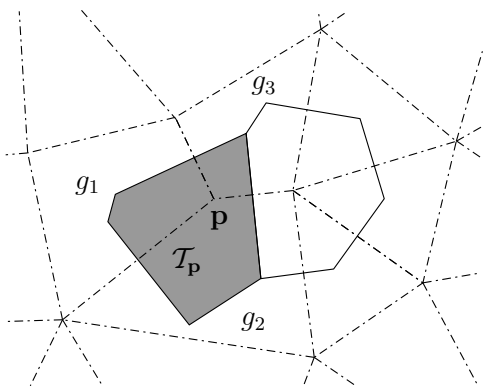

(a) $\mathcal{T}_{\mathbf{p}} \subset g_{1} \cup g_{2} \cup g_{3}$

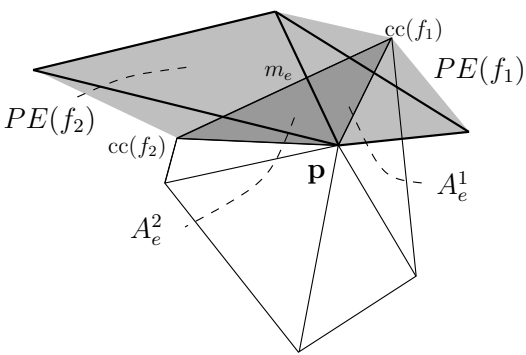

(c)

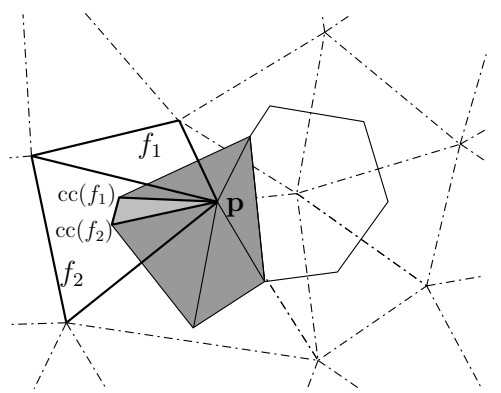

(b)

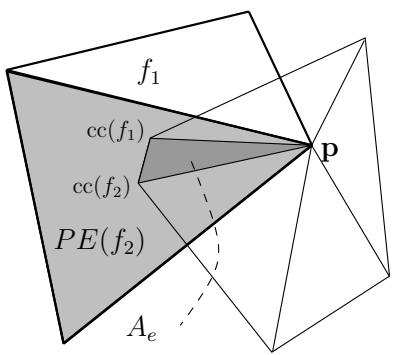

(d)

Fig. 5. Voronoi coverage of adjacent ADT tiles.

Proof. First assume that $\mathbf{p} \notin \partial \mathscr{C}(\mathbf{P})$, i.e., $\mathcal{T}_{\mathbf{p}}$ is finite as in Figure $5(\mathrm{a})$. $\mathbf{p}$ has a set of adjacent triangles $\{f\}_{f \ni \mathbf{p}}$, which in turn are adjacent via the edges $\{e\}_{e \ni \mathbf{p}}$. For each edge $e \ni \mathbf{p}$ and triangles $f_{1} \cap f_{2}=e$, we get a new triangle $A_{e}=\mathscr{C}\left(\left\{\mathbf{p}, \operatorname{cc}\left(f_{1}\right), \operatorname{cc}\left(f_{2}\right)\right\}\right)$, see Figure 5(b). This partitions $\mathcal{T}_{\mathbf{p}}$,

$$
\mathcal{T}_{\mathbf{p}}=\bigcup_{e \ni \mathbf{p}} A_{e} .
$$

So we will show that each $A_{e} \cap \mathscr{C}(\mathbf{P})$ is covered by $\mathscr{A}\left(f_{1}\right) \cup \mathscr{A}\left(f_{2}\right)$ for two cases:

$-\operatorname{cc}\left(f_{1}\right)$ and $\operatorname{cc}\left(f_{2}\right)$ are on opposite sides of $e$, see Figure 5(c): So $\operatorname{cc}\left(f_{1}\right)$ and $\operatorname{cc}\left(f_{2}\right)$ are located on the perpendicular bisector of $e$ that intersects $e$ in $m_{e}$. We can split $A_{e}$ into $A_{e}^{1}=\mathscr{C}\left(\left\{\mathbf{p}, \operatorname{cc}\left(f_{1}\right), m_{e}\right\}\right)$ and $A_{e}^{2}=\mathscr{C}\left(\left\{\mathbf{p}, \operatorname{cc}\left(f_{2}\right), m_{e}\right\}\right)$ with $A_{e}=A_{e}^{1} \cup A_{e}^{2}$. Since $m_{e}, \mathbf{p}, \operatorname{cc}\left(f_{1}\right) \in\left[P E\left(f_{1}\right)\right]$, (6) guarantees that $A_{e}^{1} \cap \mathscr{C}(\mathbf{P}) \subset \mathscr{A}\left(f_{1}\right)$ and analogously for $A_{e}^{2}$.

- $\operatorname{cc}\left(f_{1}\right)$ and $\operatorname{cc}\left(f_{2}\right)$ are on the same side of or on $e$, see Figure $5(\mathrm{~d})$ : Without loss of generality, let $f_{1}$ be obtuse. We know $\operatorname{cc}\left(f_{1}\right) \in P E\left(f_{1}\right) \backslash f_{1}$ and by (5), $\operatorname{cc}\left(f_{1}\right) \in P E\left(f_{2}\right)$. Since $\operatorname{cc}\left(f_{2}\right) \in P E\left(f_{2}\right)$ as well, $\operatorname{cc}\left(f_{1}\right), \operatorname{cc}\left(f_{2}\right), \mathbf{p} \in\left[P E\left(f_{2}\right)\right]$, and from the convexity of $\left[P E\left(f_{2}\right)\right]$ it follows that $A_{e} \cap \mathscr{C}(\mathbf{P}) \subset P E\left(f_{2}\right) \cap$ $\mathscr{C}(\mathbf{P}) \subset \mathscr{A}\left(f_{2}\right)$.

Now consider the case that $\mathbf{p} \in \partial \mathscr{C}(\mathbf{P})$, and $\mathcal{T}_{\mathbf{p}}$ becomes infinite. The argument goes as above except for the two edges $e_{1}, e_{2} \subset \partial \mathscr{C}(\mathbf{P})$ adjacent to $\mathbf{p}$ 


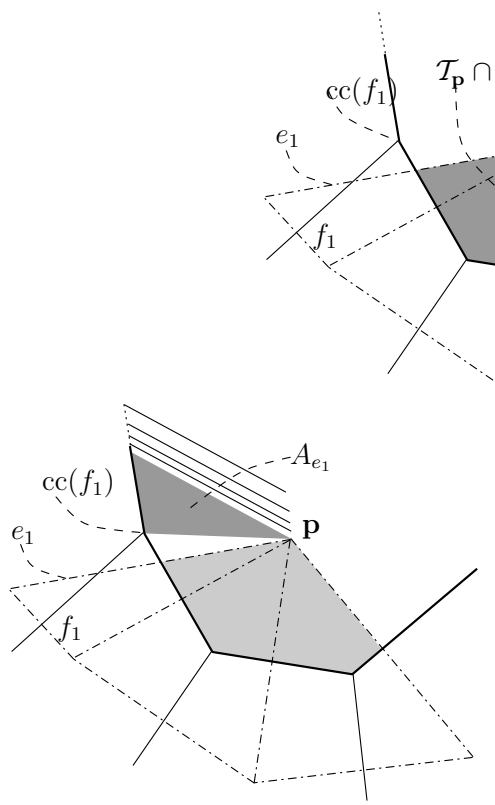

(b)

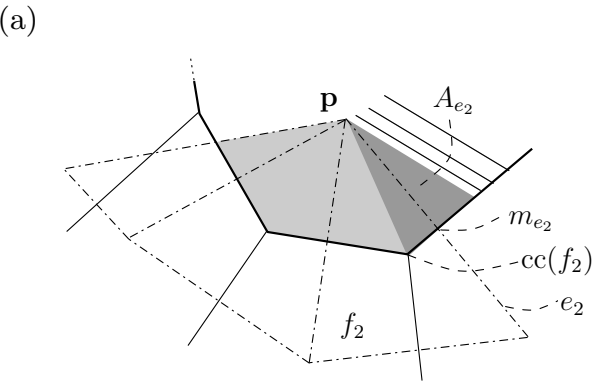

(c)

Fig. 6. Coverage of ADT tiles on the boundary of $\mathscr{C}(\mathbf{P})$. (a) For $\mathbf{p} \in \partial \mathscr{C}(\mathbf{P}), e_{1}$ and $e_{2}$ have only one adjacent triangle each. (b) Contribution of $A_{e_{1}}$ to $\mathcal{T}_{\mathbf{p}}$. (c) Contribution of $A_{e_{2}}$ to $\mathcal{T}_{\mathbf{p}}$.

which have only one adjacent triangle each, say $f_{1}$ respective $f_{2}$, as illustrated in Figure 6(a). Corresponding to the above two cases, let without loss of generality $\operatorname{cc}\left(f_{1}\right) \notin \mathscr{C}(\mathbf{P})$ like in Figure 6(b). Because $A_{e_{1}}$ is outside the convex hull, it does not contribute to $\mathcal{T}_{\mathbf{p}} \cap \mathscr{C}(\mathbf{P})$ and needs not be considered. Now, let without loss of generality $\operatorname{cc}\left(f_{2}\right) \in \mathscr{C}(\mathbf{P})$ like in Figure $6(\mathrm{c})$. The contribution of $A_{e_{2}}$ to $\mathcal{T}_{\mathbf{p}} \cap \mathscr{C}(\mathbf{P})$ is given by $\mathscr{C}\left(\mathbf{p}, m_{e_{2}}, \operatorname{cc}\left(f_{2}\right)\right) \subset f_{2} \subset \mathscr{A}\left(f_{2}\right)$.

Consequently, all parts $A_{e} \cap \mathscr{C}(\mathbf{P})$ are covered by ADT polygons, which are the covers of adjacent Delaunay triangles and thus also adjacent to $\mathbf{p}$.

\section{Discussion}

The ADT is optimal only under specific conditions, i.e., if the vertex positions cannot be changed, and the Voronoi tile of each vertex needs to be covered by adjacent polygons. A very homogeneous point set with all non-obtuse triangles is shown in Figure 7(a). In this setting, the Delaunay triangulation and the ADT are identical. Figure $7(\mathrm{~b})$ shows a point set in which ill-shaped triangles are merged into ADT polygons, thus improving the overall tile shape. Figure 7(c) demonstrates a situation where the ADT produces non-convex polygons, and the extreme case of a "hanging edge" is illustrated in Figure 7(d). Apart from these 


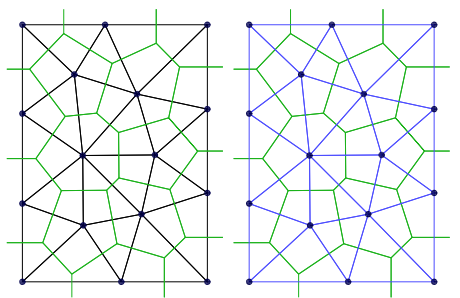

(a)

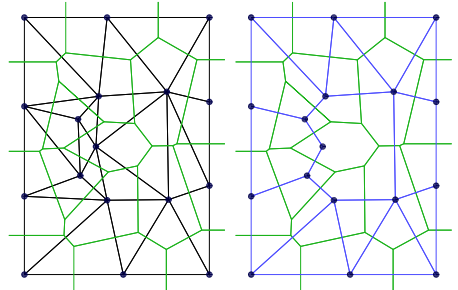

(c)

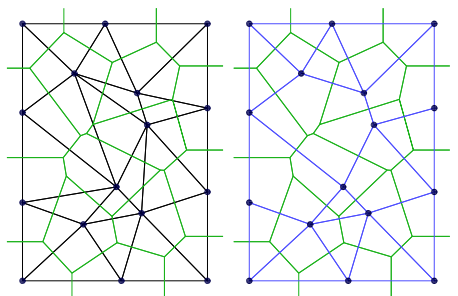

(b)

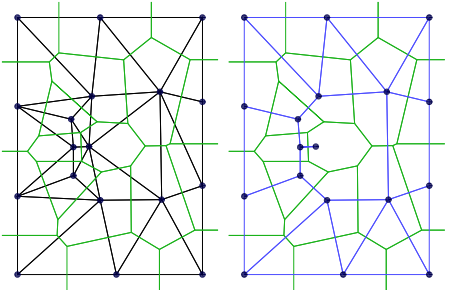

(d)

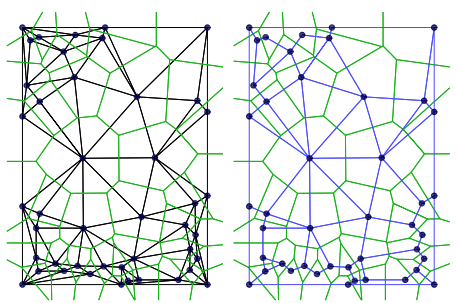

(e)

Fig. 7. Several levels of badness in the point cloud. (a) very regular, (b) some obtuse triangles, (c) constellation leading to non-convex ADT tiles, (d) constellation leading to flapping edge, (e) bad boundary elements.

artifacts inside the convex hull, which are rarely faced in practice, badly shaped polygons frequently occur near the convex hull as a result of many degenerate triangles, as illustrated in Figure 7(e). Arguably, the boundary polygons are of better shape than the triangles they are composed of. In [7], a novel polygon condition number has been introduced as a quality measure of polygonal tessellations. Extensive statistical analysis of the ADT has shown that it considerably improves the mean condition number of most unstructured triangulations over interior elements, and results in moderate improvement for boundary elements. The issues inside the convex hull can be handled by allowing vertices to move, and a regularization step could lead to more well-shaped triangles and thus better ADT-polygons. Furthermore, the boundary issues can be solved by insertion of an adequate amount of vertices along the convex hull. 


\section{Conclusion}

Certain nodal integration schemes, which are meshless extension of the Finite Element Method, lead to new requirements on the background tessellation of the domain which are no longer met by the popular Delaunay triangulation. Particularly nodal integration schemes based on the Voronoi diagram of the domain vertices entail the integration of an interpolant along the boundary of Voronoi tiles. This leads to a desirable property of the background tessellation referred to as the coverage of Voronoi tiles. To this end, the Adaptive Delaunay Tessellation for planar domains has been introduced before, but no rigorous proofs have been given.

In this paper we have introduced a simple, constructive definition of the Adaptive Delaunay Tessellation based on the Delaunay triangulation of a point set, and showed that it is unique, connected, and has the coverage of Voronoi tiles property. We furthermore provided a critical assessment of some shortcomings of the ADT that need to be considered in applications. This provides a profound theoretical background for the application of this new tessellation for nodal integration schemes in the Finite Element Method.

\section{Acknowledgements}

This work was supported by the international graduate school DFG grant 1131 on "Visualization of Large and Unstructured Data Sets - Applications in Geospatial Planning, Modeling and Engineering".

\section{References}

1. Shewchuk, J.: What is a good linear element? Interpolation, conditioning, and quality measures. In: Proc. Eleventh Int. Meshing Roundtable (Ithaca, New York), Sandia National Laboratories (2002) 115-126

2. Lohner, R.: Progress in grid generation via the advancing front technique. Eng. with Computers 12 (1996) 186-210

3. Tournois, J., Alliez, P., Devillers, O.: Interleaving delaunay refinement and optimization for 2D triangle mesh generation. In: Proc. 16th Int. Meshing Roundtable. (2007)

4. Bossen, F., Heckbert, P.: A pliant method for anisotropic mesh generation. In: Proc. 5th Int. Meshing Roundtable. (1996) 63-74

5. Antani, L., Delage, C., Alliez, P.: Mesh sizing with additively weighted voronoi diagrams. In: Proc. 16th Int. Meshing Roundtable. (2007)

6. Hjelle, Ø., Dæhlen, M.: Triangulations and Applications. Mathematics and Visualization. Springer, Secaucus, NJ, USA (2006)

7. Constantiniu, A., Steinmann, P., Bobach, T., Farin, G., Umlauf, G.: The adaptive delaunay tessellation: A neighborhood covering meshing technique. Computational Mechanics 42(5) (2008) 655-669

8. Sukumar, N., Moran, B., Semenov, A., Belikov, V.: Natural neighbour Galerkin methods. Int. J. for Numerical Methods in Engineering 50 (2001) 1-27

9. Chen, J., Wu, C., Yoon, S., You, Y.: A stabilized conforming nodal integration for Galerkin mesh-free methods. Int. J. Numer. Methods Eng. 50 (2001) 435-466 